\title{
Determinação de valores próprios de referência para vitamina $D$, hormônio paratireoideo e cálcio em um laboratório de análises clínicas
}

\author{
Determination of proper reference intervals for vitamin $D$, parathyroid hormone \\ and calcium in a clinical laboratory
}

\author{
Adriana Dalpicolli Rodrigues $(\mathbb{D})^{1,2} \bowtie$, Estefânia Weirich $(\mathbb{D})$, Eunice Zanandréa Duarte (iD) 1 \\ ${ }^{1}$ Laboratório Alfa Ltda. Caxias do Sul, RS. \\ 2 Centro Universitário e Faculdades Úniftec. Caxias do Sul, RS. \\ Parte deste trabalho foi apresentado ao 440 Congresso Brasileiro de Análises Clínicas, João Pessoa/PB, junho de 2017 e \\ ao 51ํ Congresso Brasileiro de Patologia Clínica, São Paulo/SP, setembro de 2017.
}

Como citar este artigo (How to cite this article):

Rodrigues AD, Weirich E, Duarte EZ. Determinação de valores próprios de referência para vitamina $\mathrm{D}$, hormônio paratireoideo e cálcio em um laboratório de análises clínicas (Determination of proper reference intervals for vitamin D, parathyroid hormone and calcium in a clinical laboratory). Sci Med. 2018;28(4):ID28462. DOI: 10.15448/1980-6108.2018.4.28462

\section{RESUMO}

OBJETIVOS: Determinar os valores próprios de referência para vitamina D, hormônio paratireoideo (PTH) e cálcio em um laboratório de análises clínicas.

MÉTODOS: Foi realizado um estudo transversal retrospectivo nas unidades do Laboratório Alfa Ltda., localizado em Caxias do Sul, na região serrana do Rio Grande do Sul. A amostra incluiu clientes e funcionários do laboratório, que se autodeclaravam saudáveis. O estudo foi baseado em dois métodos de pesquisa (direto e indireto) propostos pelo Clinical \& Laboratory Standards Institute para a determinação de VR próprios de um laboratório. O método direto (MD) envolveu aplicação de questionário, coleta e análise de amostras de sangue e o método indireto (MI) consistiu em análise de banco de dados.

RESULTADOS: No MD foram avaliados resultados de 241 indivíduos, sendo 120 do gênero feminino. A média de idade dos participantes foi de 33,8 $\pm 10,6$ anos. O MI resultou em 5.485 valores para as dosagens de vitamina D, 191 para o PTH e 856 para o cálcio. A média de idade dos

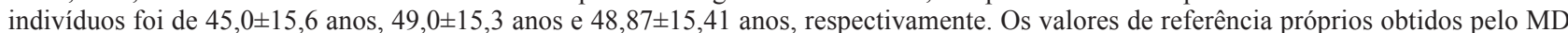
foram estatisticamente iguais aos do MI para as três dosagens. Quando comparados os valores de referência obtidos com os disponibilizados pelo fabricante, a vitamina D resultou em uma faixa mais baixa de normalidade (fabricante: $>30 \mathrm{ng} / \mathrm{mL} ; \mathrm{MD}: 10-47 \mathrm{ng} / \mathrm{mL} ; \mathrm{MI}$ : $11-46 \mathrm{ng} / \mathrm{mL}$ ), o PTH em uma faixa maior (fabricante: 15-68,3 ng/mL; MD: 19,2-81,6 ng/mL; MI: 26,1-94,3 ng/mL) e o cálcio apresentou valores muito próximos (fabricante: $8,3-10,6 \mathrm{ng} / \mathrm{mL}$; MD: 8,3-10,2 ng/mL; MI: 8,2-10,1 ng/mL). Houve diferença estatística entre os valores de referência estabelecidos para vitamina $\mathrm{D}$ e cálcio entre os gêneros.

CONCLUSÕES: Tanto o MD quanto o MI foram eficientes para a determinação de valores de referência. Os valores de referência estabelecidos no estudo, especificamente para a vitamina D e o PTH, foram diferentes dos sugeridos nas instruções do fabricante, o que reforça a recomendação de estabelecimento de valores de referência próprios para cada laboratório.

DESCRITORES: valores de referência; intervalos de referência; testes laboratoriais; saúde da população.

\section{ABSTRACT}

AIMS: To determine the proper reference intervals for vitamin D, parathyroid hormone (PTH) and calcium in a clinical laboratory.

METHODS: A retrospective cross-sectional study was carried out at the units of Alfa Ltda. Laboratory, located in Caxias do Sul, in the mountain region of Rio Grande do Sul. The sample included self-declared healthy clients and laboratory workers. The study was based on two research methods (direct and indirect) proposed by the Clinical \& Laboratory Standards Institute for the establishment of proper reference intervals for a specific laboratory. The direct method (DM) involved questionnaire application, collection and analysis of blood samples and the indirect method (IM) consisted of database analysis.

RESULTS: Results of 241 individuals were evaluated in the DM, of which 120 were female. The mean age of participants was $33.8 \pm 10.6$ years. IM resulted in 5,485 values for vitamin D, 191 for PTH, and 856 for calcium. The mean age of the individuals was $45.0 \pm 15.6$ years, $49.0 \pm 15.3$ years and $48.87 \pm 15.41$ years, respectively. The proper reference intervals obtained by the DM were statistically equal to those of the IM for the three dosages. When comparing the reference intervals obtained with those available in the manufacturer report, vitamin $\mathrm{D}$ resulted 
in a lower range of normality (report: $>30 \mathrm{ng} / \mathrm{mL}$; MD: $10-47 \mathrm{ng} / \mathrm{mL}$; MI: $11-46 \mathrm{ng} / \mathrm{mL}$ ), PTH in a higher range (report: $15-68.3 \mathrm{ng} / \mathrm{mL}$; MD: $19.2-81.6 \mathrm{ng} / \mathrm{mL}$; MI: 26.1-94.3 ng/mL), and calcium presented very close values (report: $8.3-10.6 \mathrm{ng} / \mathrm{mL}$; MD: $8.3-10.2 \mathrm{ng} / \mathrm{mL}$; MI: $8.2-10.1 \mathrm{ng} / \mathrm{mL}$ ). There was a statistical difference between the reference intervals established for vitamin D and calcium between genders.

CONCLUSIONS: Both MD and MI were efficient for the determination of proper reference intervals. The reference intervals established in the study, specifically for vitamin D and PTH, were different from those suggested in the manufacturer's package insert, which reinforces the recommendation of establishing proper reference intervals for each laboratory.

KEYWORDS: reference values; reference range; laboratory test; population health.

Abreviaturas: CLSI, Clinical \& Laboratory Standards Institute; MD, método direto de pesquisa; MI, método indireto de pesquisa; PALC, Programa de Acreditação de Laboratórios Clínicos; $\mathrm{PTH}$, hormônio paratireoideo (parathyroid hormone); VR, valor(es) de referência.

\section{INTRODUÇÃO}

Os valores de referência (VR) presentes em um laudo emitido por um laboratório de análises clínicas representam o intervalo onde a grande maioria da população saudável está inserida. Essa ferramenta auxilia o médico na tomada de decisões para diferenciar pacientes doentes dos saudáveis, facilitando a determinação do diagnóstico correto e a decisão terapêutica mais adequada para cada caso [1].

Não há nenhuma lei no Brasil que exija a determinação de VR próprios para os laboratórios de análises clínicas. Desse modo, normalmente os valores utilizados são preconizados pelos fabricantes dos reagentes ou descritos na literatura, muitas vezes internacional ou ultrapassada, sem um estudo da população em questão [2].

O Clinical \& Laboratory Standards Institute (CLSI), dos Estados Unidos da América, recomenda que cada laboratório estabeleça seus próprios VR, pois cada população possui indivíduos com diferentes características fisiológicas, condições ambientais, estilo de vida e hábitos alimentares. O CLSI preconiza que essa determinação pode ser feita por métodos diretos, através de avaliação de analitos em indivíduos saudáveis, ou indiretos, pela consulta de resultados em banco de dados [1]. O Programa de Acreditação de Laboratórios Clínicos (PALC) no requisito $9.8 \mathrm{da}$ Norma 2016, preconiza que o laboratório deve verificar se os VR informados pelo fabricante são adequados à população atendida [3].

Há estudos que mostram que caucasianos possuem maior facilidade de síntese da vitamina D (25-Hidroxivitamina D) por exposição solar quando comparados a afrodescendentes, entretanto, em latitudes mais distantes da zona tropical, a radiação torna-se menor e o uso de maior quantidade de vestimentas dificulta o processo de exposição ao sol [4-7]. A síntese de vitamina $\mathrm{D}$ pelo organismo, que ocorre pela irradiação solar sobre a pele, é de fundamental importância para o metabolismo ósseo [4, 6]. A relação da vitamina D com o hormônio paratireoideo (PTH) é inversa, havendo inflexão perto dos $20-30 \mathrm{ng} / \mathrm{mL}$ de vitamina sérica. A má absorção do cálcio não ocorre até valores graves de deficiência de vitamina $\mathrm{D}$, devido à resposta do $\mathrm{PTH}$, com consequente manutenção dos níveis da forma ativa da vitamina $\mathrm{D}(1,25-\mathrm{OH}-2$ Vitamina D) [8].

O presente estudo teve por objetivo determinar os VR próprios do laboratório, com método direto (MD) e método indireto (MI) de pesquisa, para a Vitamina D, PTH e cálcio, tendo em vista o tipo de população da região.

\section{MÉTODOS}

Foi realizado um estudo transversal retrospectivo nas unidades do Laboratório Alfa Ltda., maior laboratório de análises clínicas do interior do Rio Grande do Sul, localizado em Caxias do Sul, certificado pelo PALC e pela ISO. A cidade pertence à região nordeste do estado, com elevada altitude e clima temperado $[9,10]$. Segundo o IBGE [9], grande parte da população desse município é de imigração europeia, com características de pele branca, e consequentemente mais sensível à exposição solar.

O estudo foi aprovado pelo Comitê de Ética em Pesquisa Sociedade Educacional Santa Rita Ltda., Faculdade da Serra Gaúcha, sob o parecer consubstanciado de número 1.256.237. O estudo foi realizado em duas etapas, entre março de 2015 e setembro de 2016, seguindo as normas preconizadas pelo CLSI [1].

Primeira etapa: realização do MD. Foram selecionados, entre clientes e funcionários do laboratório, indivíduos que se autodeclararam saudáveis, com 
18 anos ou mais, aceitaram participar da pesquisa e assinaram o termo de consentimento livre e esclarecido. Na mesma ocasião da assinatura do termo, os voluntários responderam a um questionário adaptado do CLSI [1] sobre sua saúde. O número amostral foi o preconizado pelo CLSI [1] (mínimo de 120 indivíduos). A coleta da amostra biológica seguiu padrões convencionais aplicados na rotina laboratorial. A forma de seleção foi a priori, ou seja, após avaliação do questionário, os pacientes que se autodeclararam saudáveis foram selecionados e as dosagens de Vitamina D, PTH e cálcio foram realizadas nos respectivos setores de análise do laboratório, conforme procedimento operacional padrão e normas descritas pelo fabricante de cada equipamento. $\mathrm{Na}$ primeira etapa foram excluídos do estudo indivíduos fumantes, alcoolistas, obesos classificados através do índice de massa corporal (IMC) pelos critérios da Organização Mundial da Saúde (IMC $\geq 30 \mathrm{~kg} / \mathrm{m}^{2}$ ), gestantes, pacientes que haviam realizado cirurgia há menos de 6 meses, que não moravam na mesma região do estudo e que usavam drogas ou medicamentos/suplementos alimentares, como por exemplo, vitamina $\mathrm{D}$ e cálcio, que pudessem interferir nas análises laboratoriais. Também foram excluídos pacientes que não responderam completamente ao questionário.

Segunda etapa: realização do MI, baseado na consulta dos resultados de Vitamina D, PTH e cálcio no banco de dados do Laboratório Alfa Ltda. Não foi aplicado questionário, mas avaliaram-se os resultados dos demais exames realizados pelo indivíduo no mesmo período, excluindo os que apresentaram resultados fora da normalidade ou informações contidas no cadastro do paciente que poderiam comprometer o estudo, como a medicação em uso. O número amostral foi por conveniência. Na segunda etapa foram excluídos os pacientes que apresentaram resultados de exames alterados de perfil renal, hepático e da tireoide, uso de suplementos, cálcio, vitamina D e medicamentos para tratamento da tireoide, a partir de avaliação de informações contidas no banco de dados.

Foram constituídas tabelas com valores mínimos e máximos, média \pm desvio padrão (DP) e VR obtidos segundo o CLSI [1]. Calculou-se a frequência de indivíduos com valores fora da normalidade para os três parâmetros. Realizou-se também o teste qui-quadrado para comparar os resultados obtidos via MD e MI e o teste $t$ de Student para comparação das médias das dosagens por gênero. A análise de dados foi realizada por meio do programa SPSS 20.0 para Windows com níveis de significância de $\mathrm{p}<0,05$.

\section{RESULTADOS}

Foi aplicado questionário a 276 indivíduos (146 mulheres) para determinação de VR a partir do MD durante os meses de primavera/verão (novembro de 2015 a março de 2016) e outono/inverno (abril de 2016 a agosto 2016). Desse total, foram excluídos nove indivíduos do gênero masculino e 26 do gênero feminino a partir dos critérios de exclusão. Nos demais (total de 241, sendo 120 pertencentes ao gênero feminino) foram realizadas as dosagens de Vitamina D, PTH e cálcio. A média de idade dos participantes foi de $33,8 \pm 10,6$ anos, com idade mínima de 18 e a máxima de 66 anos, sendo caucasiana a etnia prevalente autodeclarada (94,2\% das mulheres e $93,4 \%$ dos homens).

A avaliação do banco de dados de março a julho de 2015 para realização do MI resultou em 9.842 valores para a vitamina $\mathrm{D}$, sendo excluídos 4.357; 444 resultados para o $\mathrm{PTH}$, sendo excluídos 253; e 1.631 para o cálcio sendo excluídos 775. A média de idade dos indivíduos foi de 45, $0 \pm 15,6$ anos para Vitamina D, $49,0 \pm 15,3$ anos para o PTH e $48,9 \pm 15,4$ anos para o cálcio. A idade mínima foi de 18 anos para todas as avaliações e a idade máxima de 97,90 e 92 anos, respectivamente. Não foi possível avaliar a etnia no banco de dados, devido à ausência dessa informação.

Observou-se que os VR próprios obtidos pelo MD foram muito próximos aos observados no MI para as três dosagens avaliadas, não apresentando diferença estatística. Do mesmo modo, não foi observada diferença estatística nas avaliações por gênero para PTH. Já para vitamina D e cálcio observou-se diferença entre os gêneros em ambos os métodos (Tabela 1).

Em relação às estações do ano, verificou-se pelo MD que a média de vitamina $\mathrm{D}$ foi maior no verão $(31,8 \pm 9,9 \mathrm{ng} / \mathrm{dL})$ e menor no inverno $(19,5 \pm 8,1 \mathrm{ng} / \mathrm{dL})$.

Segundo os VR próprios obtidos para a vitamina D neste estudo, devem ser considerados normais valores que no laudo estariam na faixa de deficiência $(21,2 \%$ pelo MD e $19,1 \%$ pelo $\mathrm{MI}$ ) e insuficiência (40,2\% pelo $\mathrm{MD}$ e $50,9 \%$ pelo $\mathrm{MI})$. Em relação ao $\mathrm{PTH}$, apenas $8,3 \%(7,5 \%$ com vitamina $\mathrm{D} \leq 30 \mathrm{ng} / \mathrm{mL})$ dos indivíduos avaliados pelo MD e 14,7\% (todos com vitamina $D$ abaixo de $13 \mathrm{ng} / \mathrm{mL}$ ) pelo MI apresentaram valores acima do estabelecido no laudo. Entretanto, se levarmos em consideração o VR próprio obtido, a faixa de normalidade é maior, resultando em um percentual menor de indivíduos com PTH alto. Já o VR próprio estabelecido para o cálcio apresentou valores muito próximos aos disponibilizados no laudo. O percentual de indivíduos com cálcio inferior a normalidade foi menor do que $3 \%$ em ambos os métodos. 
Tabela 1. Resultados e valores de referência de vitamina D, hormônio paratireoideo e cálcio de indivíduos que vivem na região serrana do sul do Brasil, por gênero, avaliados a partir do método direto e indireto entre 2015 e 2016.

\begin{tabular}{|c|c|c|c|c|c|c|c|}
\hline Exame/método & $\begin{array}{l}\text { Gênero } \\
\text { (n) }\end{array}$ & $\begin{array}{c}\text { Valor } \\
\text { mínimo-máximo }\end{array}$ & Média $\pm D P$ & $\begin{array}{l}\text { VR próprio } \\
\text { obtido }\end{array}$ & $\begin{array}{l}\text { Valor de } p \\
\text { Comparação de } \\
\text { métodos }\end{array}$ & $\begin{array}{l}\text { Valor de } p \\
\text { Comparação de } \\
\text { gêneros }\end{array}$ & VR do fabricante \\
\hline $\begin{array}{l}\text { Vitamina } \mathrm{D}(\mathrm{ng} / \mathrm{mL}) / \\
\text { Método direto }\end{array}$ & $\begin{array}{l}A(241) \\
F(120) \\
M(121)\end{array}$ & $\begin{array}{c}<10-93 \\
<10-93 \\
10-62\end{array}$ & $\begin{array}{c}28,3 \pm 10,4 \\
24,4 \pm 10,9 \\
32,1 \pm 8,4\end{array}$ & $\begin{array}{l}10-47 \\
10-43 \\
14-49\end{array}$ & & $<0,001$ & $\begin{array}{l}\text { Deficiência: }<20 \\
\text { Insuficiência: } 20 \text { a } 30 \\
\text { Suficiência: } 30 \text { a } 100 \\
\text { Excesso: } 100 \text { a } 150 \\
\text { Intoxicação: > } 150\end{array}$ \\
\hline $\begin{array}{l}\text { Vitamina } \mathrm{D}(\mathrm{ng} / \mathrm{mL}) / \\
\text { Método indireto }\end{array}$ & $\begin{array}{l}A(5.485) \\
F(4.694) \\
M(791)\end{array}$ & $\begin{array}{l}4-127 \\
4-127 \\
7-102\end{array}$ & $\begin{array}{l}26,8 \pm 9,0 \\
26,5 \pm 9,0 \\
28,6 \pm 9,2\end{array}$ & $\begin{array}{l}11-46 \\
10-45 \\
13-48\end{array}$ & 0,060 & $<0,001$ & \\
\hline $\begin{array}{l}\text { PTH }(\mathrm{pg} / \mathrm{mL}) / \\
\text { Método direto }\end{array}$ & $\begin{array}{l}A(241) \\
F(120) \\
M(121)\end{array}$ & $\begin{array}{l}13,2-99,8 \\
18,2-99,8 \\
13,2-90,2\end{array}$ & $\begin{array}{l}44,3 \pm 16,4 \\
43,7 \pm 16,9 \\
44,9 \pm 16,0\end{array}$ & $\begin{array}{l}19,2-81,6 \\
19,5-89,0 \\
17,6-81,6\end{array}$ & & 0,576 & 15,0 a 68,3 \\
\hline $\begin{array}{l}\text { PTH }(\mathrm{pg} / \mathrm{mL}) / \\
\text { Método indireto }\end{array}$ & $\begin{array}{l}A(191) \\
F(128) \\
M(63)\end{array}$ & $\begin{array}{l}17,5-133,0 \\
23,4-133,0 \\
17,5-131,8\end{array}$ & $\begin{array}{l}48,9 \pm 19,7 \\
50,3 \pm 20,0 \\
46,0 \pm 19,0\end{array}$ & $\begin{array}{l}26,1-94,3 \\
26,2-95,0 \\
19,9-83,4\end{array}$ & 0,365 & 0,155 & \\
\hline $\begin{array}{l}\text { Cálcio }(\mathrm{mg} / \mathrm{dL}) / \\
\text { Método direto }\end{array}$ & $\begin{array}{l}A(241) \\
F(120) \\
M(121)\end{array}$ & $\begin{array}{l}8,0-10,7 \\
8,0-10,3 \\
8,0-10,7\end{array}$ & $\begin{array}{l}9,1 \pm 0,5 \\
9,0 \pm 0,4 \\
9,2 \pm 0,5\end{array}$ & $\begin{array}{l}8,3-10,2 \\
8,3-10,0 \\
8,2-10,3\end{array}$ & & 0,001 & 8,3 a 10,6 \\
\hline $\begin{array}{l}\text { Cálcio }(\mathrm{mg} / \mathrm{dL}) / \\
\text { Método indireto }\end{array}$ & $\begin{array}{l}A(856) \\
F(705) \\
M(151)\end{array}$ & $\begin{array}{l}7,7-11,9 \\
7,7-11,9 \\
7,9-11,9\end{array}$ & $\begin{array}{l}9,0 \pm 0,5 \\
9,0 \pm 0,5 \\
9,1 \pm 0,5\end{array}$ & $\begin{array}{l}8,2-10,1 \\
8,2-10,1 \\
8,3-10,2\end{array}$ & 0,618 & 0,008 & \\
\hline
\end{tabular}

DP, desvio padrão; VR, valor de referência; A, ambos os gêneros; F, gênero feminino; M, gênero masculino; PTH, hormônio tireoideo.

\section{DISCUSSÃO}

Com o presente estudo foi possível observar que a média geral de vitamina $\mathrm{D}$ da população avaliada foi muito próxima em ambos os métodos de pesquisa (MD e MI). Mais da metade dos indivíduos apresentaram níveis de vitamina $\mathrm{D}$ deficientes ou insuficientes segundo os VR sugeridos na bula do fabricante do reagente para determinação desse analito e Holick et al. [11] (deficientes inferior a $20 \mathrm{ng} / \mathrm{mL}$ e insuficientes de 21 a $29 \mathrm{ng} / \mathrm{mL}$ ). Segundo Rosen [12], utilizando a faixa de VR inferior a $29 \mathrm{ng} / \mathrm{mL}$, a prevalência estimada de insuficiência de vitamina D é de 50 a $80 \%$ na população geral e depende de vários fatores para a manutenção dos níveis séricos, como as estações do ano, exposição solar, latitude, alimentação, entre outros.

Em uma revisão de Maeda et al. [13], em estudos realizados em várias regiões do Brasil, foi indicada alta prevalência de hipovitaminose $\mathrm{D}$ em diversas faixas etárias, principalmente em idosos. Há poucos estudos que avaliaram os níveis de vitamina $\mathrm{D}$ na população saudável no Rio Grande do Sul. Em uma pesquisa realizada na cidade de Farroupilha $(n=296)$ foram encontrados resultados semelhantes aos nossos, onde $75 \%$ dos avaliados possuíam valores deficientes ou insuficientes de vitamina D [14]. Em um grupo de médicos de Porto Alegre, 57,4\% apresentaram resultados de vitamina $\mathrm{D}$ na faixa de deficiente (inferior a $20 \mathrm{ng} / \mathrm{mL}$ ) [4].

Seguindo as normas do CLSI [1] para determinar os VR próprios do laboratório e como solicitado pelo PALC [3], foi observado que os indivíduos com dosagens de vitamina $D$ entre 10 e $47 \mathrm{ng} / \mathrm{mL}$ estão dentro da normalidade. Considerando os VR determinados por este estudo, menos de $0,5 \%$ da população estudada estaria no estado de deficiência para essa vitamina. Esses VR estão muito próximos aos estabelecidos pelo Institute of Medicine, dos Estados Unidos da América, o qual determina como deficiência valores inferiores a $12 \mathrm{ng} / \mathrm{mL}$ e como limite superior $50 \mathrm{ng} / \mathrm{mL}[15]$.

Há autores que definem o limite inferior do intervalo normal de vitamina $\mathrm{D}$ em $30 \mathrm{ng} / \mathrm{mL}$, sob alegação de que abaixo desse valor os níveis de PTH se elevam e a absorção ativa de cálcio é comprometida $[8,12]$. Entretanto, em nosso estudo observamos que apenas $8,3 \%$ dos indivíduos avaliados pelo MD e 14,7\% pelo MI apresentaram valores de PTH acima do estabelecido no laudo, contra um percentual muito maior de vitamina $\mathrm{D}$ inferior a $30 \mathrm{ng} / \mathrm{mL}$ e cálcio dentro da normalidade em mais de $97 \%$ dos casos. Rosen [12] relata que há muitos estudos inconsistentes em relação à associação dos níveis de vitamina $\mathrm{D}, \mathrm{PTH}$ e cálcio, e também sobre os danos ósseos causados em situações de deficiência/insuficiência da vitamina D. 
Eloi et al. [16], em São Paulo, obtiveram resultados muito próximos aos nossos, onde $70 \%$ dos indivíduos avaliados apresentaram deficiência ou insuficiência de vitamina $\mathrm{D}$ (média 25,6 $\pm 11,4 \mathrm{ng} / \mathrm{mL}$ ) com valor médio de PTH de $44,6 \pm 23,6 \mathrm{pg} / \mathrm{mL}$ e de cálcio $9,4 \pm 0,5 \mathrm{mg} / \mathrm{dL}$. Diferentemente deste estudo, esses autores não observaram diferença estatística entre os gêneros nas avaliações realizadas. Eles sugerem que essa igualdade pode ser devida ao fato da exposição à luz solar entre os gêneros ser a mesma na população estudada. Em contrapartida, em nosso estudo, acreditamos que provavelmente a exposição não seja a mesma, pois o Rio Grande do Sul, principalmente nas regiões mais altas, apresenta temperaturas mais baixas e geralmente as mulheres utilizam mais roupas do que os homens para manter a temperatura corporal.

Em um estudo realizado na Turquia foram considerados como suficientes níveis de vitamina $\mathrm{D}$ superiores a $23,5 \mathrm{ng} / \mathrm{mL}$, insuficientes entre 15,7 e $23,6 \mathrm{ng} / \mathrm{mL}$ e deficientes quando inferiores a $15,7 \mathrm{ng} / \mathrm{mL}$. Mesmo utilizando uma faixa mais baixa de VR disponíveis na literatura, aquele estudo também obteve alto percentual de deficiência de vitamina D, com $75 \%$ dos resultados avaliados. O PTH mostrou elevação com valores de vitamina $D$ inferior a $23,6 \mathrm{ng} / \mathrm{dL}$, considerando esse limite como normalidade para a vitamina. Os autores observaram diferença estatística por gênero, corroborando com nosso estudo [17]. O trabalho ainda ressalta a importância de determinação de VR para a vitamina D e PTH por gênero e sazonalidade, assim como também sugerido por outros estudos [7, 16-18].

Em 2018 foi divulgada pela Sociedade Brasileira de Patologia Clínica e Medicina Laboratorial um posicionamento sobre os intervalos de referência para vitamina D que foi elaborado juntamente com a Sociedade Brasileira de Endocrinologia e Metabolo- gia [19]. Segundo o documento, abaixo de $10 \mathrm{ng} / \mathrm{mL}$ são níveis muito baixos, acima de $20 \mathrm{ng} / \mathrm{mL}$ é o valor desejável para população saudável (até 60 anos), entre 30 e $60 \mathrm{ng} / \mathrm{mL}$ é o valor recomendado para grupos de risco (idosos, gestantes, lactantes, doenças ósseas, inflamatórias, autoimunes, etc.) e acima de $100 \mathrm{ng} / \mathrm{mL}$ há risco de toxicidade e hipercalcemia [19]. Consideram-se esses valores mais próximos aos observados na nossa pesquisa.

Pelas características da região (elevada altitude, clima frio, menor incidência solar), os níveis de vitamina $\mathrm{D}$ na população estudada são semelhantes aos que ocorrem nos meses mais frios em regiões que apresentam nítida variação sazonal, entretanto diferentes de locais de clima tropical [7, 16-18]. Desse modo, reforça-se a recomendação de estabelecimento de VR próprios para cada laboratório. Com o presente estudo, foi possível estabelecer VR próprios para as dosagens de vitamina $\mathrm{D}, \mathrm{PTH}$ e cálcio, pelo MD e MI de pesquisa, de indivíduos do sul do Brasil atendidos no Laboratório Alfa Ltda.

\section{NOTAS}

Apoio financeiro

Este estudo não recebeu apoio financeiro de fontes externas.

Declaração de conflito de interesses

Os autores declaram não haver conflitos de interesses relevantes ao conteúdo deste estudo.

Contribuições dos autores

Todos os autores fizeram contribuições substanciais para concepção, ou delineamento, ou aquisição, ou análise ou interpretação de dados; e redação do trabalho ou revisão crítica; e aprovação final da versão para publicação.

Disponibilidade dos dados e responsabilidade pelos resultados

Todos os autores declaram ter tido total acesso aos dados obtidos e assumem completa responsabilidade pela integridade destes resultados.

\section{REFERÊNCIAS}

1. CLSI - Clinical and Laboratory Standards Institute. Defining, Establishing, and Verifying Reference Intervals in the Clinical Laboratory; Approved Guideline. $3^{\text {rd }}$ ed. CLSI document C28-A3. Wayne, PA: Clinical and Laboratory Standards Institute; 2008.

2. BRASIL. Ministério da Saúde. Agência Nacional de Vigilância Sanitária (ANVISA). Dispõe sobre regulamentação técnica para funcionamento de laboratórios clínicos. Resolução da Diretoria Colegiada - RDC no 302, 2005.

3. Chaves CD, Ferreira CES, Berlitz FA, Oliveira GF, Villela LHC, Bastos RV, Pereira R, Shcolnik W. Sociedade Brasileira de Patologia Clínica (SBPC/ML). Programa de Acreditação de Laboratórios Clínicos (PALC) - Norma 2016 [Internet]. Rio de Janeiro; 2016 [updated 2016; cited 2017 fevereiro 20]. Available from: http://www.sbpc.org.br/upload/conteudo/ norma_palc_2016_web.pdf

4. Premaor MO, Paludo P, Manica D, Paludo AP, Rossatto ER, Scalco R, Furlanetto TW. Hypovitaminosis D and secondary hyperparathyroidism in resident physicians of a general hospital in southern Brazil. J Endocrinol Invest. 2008;31(11): 991-5. https://doi.org/10.1007/BF03345637 
5. Fragoso TS, Dantas AT, Marques CDL, Rocha Junior LF, Melo JHL, Costa AJG, Duarte ALBP. Níveis séricos de 25 hidroxivitamina D3 e sua associação com parâmetros clínicas e laboratoriais em pacientes com lúpus eritematoso sistêmico. Rev Bras Reumatol. 2012;52(1):60-5. https://doi.org/10.1590/S0482-50042012000100007

6. Nowson CA, McGrath JJ, Ebeling PR, Haikerwal A, Daly RM, Sanders KM, Seibel MJ, Mason RS. Vitamin D and health in adults in Australia and New Zealand: a position statement. Med J Aust. 2012;196(11):686-7. https://doi.org/10.5694/ mja11.10301

7. Darling AL, Hart KH, Gibbs MA, Gossiel F, Kantermann T, Horton K, Johnsen S, Berry JL, Skene DJ, Eastell R, Vieth R, Lanham-New SA. Greater seasonal cycling of 25-hydroxy vitamin D is associated with increased parathyroid hormone and bone resorption. Osteoporos Int. 2014;25:933-41. https://doi.org/10.1007/s00198-013-2493-4

8. Alves M, Bastos M, Leitão F, Marques G, Ribeiro G, Carrilho F. Vitamina D-importância da avaliação laboratorial. Rev Port Endocrinol, Diabetes Metabol. 2013;8(1):32-9. https://doi.org/10.1016/j.rpedm.2012.12.001

9. Brasil. Instituto Brasileiro de Geografia e Estatística - IBGE. Características Étnico-Raciais da População. Um estudo das categorias de classificação de cor ou raça 2008 [Internet]. Rio de Janeiro; 2011 [updated 2015; cited 2018 Jan 19]. Available from: www.ibge.gov.br/home/estatistica/populacao/caracteristicas_raciais/PCERP2008.pdf.

10. Empresa Brasileira de Pesquisa Agropecuária - Embrapa. Atlas climático da região sul do Brasil, Estados do Paraná, Santa Catarina e Rio Grande do Sul [Internet]. Brasília, DF; 2012. [Updated 2015; cited 2018 Jan 20]. Available from: https:/www.embrapa.br/busca-de-publicacoes/-/publicacao/1045852/atlas-climatico-da-regiao-sul-do-brasil-estados-doparana-santa-catarina-e-rio-grande-do-sul.

11. Holick MF, Binkley NC, Bischoff-Ferrari HA, Gordon CM, Hanley DA, Heaney RP, Murad MH, Weaver CM; Endocrine Society. Evaluation, Treatment, and Prevention of Vitamin D Deficiency: an Endocrine Society Clinical Practice Guideline. J Clin Endocrinol Metab. 2011;96(7):1911-30. https://doi.org/10.1210/jc.2011-0385

12. Rosen CJ. Vitamin D insufficiency. N Engl J Med. 2011;364(3):248-54. https://doi.org/10.1056/NEJMcp1009570

13. Maeda SS, Borba VZC, Camargo MBR, Silva DMW, Borges JLC, Bandeira Fk, Lazaretti-Castro M. Recomendações da Sociedade Brasileira de Endocrinologia e Metabologia (SBEM) para o diagnóstico e tratamento da hipovitaminose D. Arq Bras Endocrinol Metab. 2014;58(5):411-33. https://doi.org/10.1590/0004-2730000003388

14. Moraes BF, Barbora FAT, Cenci RP, Martins TL, Poeta J. Prevalência da carência de vitamina D sérica em moradores de Farroupilha/RS, Brasil, 2013. In: Anais II Cong Pesq Ext da FSG. 2014;2(2):8269.

15. Aloia JF. Clinical Review: The 2011 report on dietary reference intake for vitamin D: where do we go from here? J Clin Endocrinol Metab. 2011;96(10):2987-96. https://doi.org/10.1210/jc.2011-0090

16. Eloi M, Horvath DV, Szejnfeld VL, Ortega JC, Rocha DA, Szejnfeld J, Castro CH. Vitamin D deficiency and seasonal variation over the years in São Paulo, Brazil. Osteoporos Int. 2016;27(12):3449-56. https://doi.org/10.1007/s00198-0163670-z

17. Serdar MA, Can BB, Kilercik M, Durer ZA, Aksungar FB, Serteser M, Coskun A, Ozpinar A, Unsal I. Analysis of changes in parathyroid hormone and $25(\mathrm{oh})$ vitamin d levels with respect to age, gender and season: a data mining study. J Med Biochem. 2017;36(1):73-83. https://doi.org/10.1515/jomb-2017-0002

18. Kroll MH, Bi C, Garber CC, Kaufman HW, Liu D, Caston-Balderrama A, Zhang K, Clarke N, Xie M, Reitz RE, Suffin SC, Holick MF. Temporal relationship between vitamin D status and parathyroid hormone in the United States. PLoS One. 2015;10:e0118108. https://doi.org/10.1371/journal.pone.0118108

19. Ferreira CES, Maeda SS, Batista MC, Lazaretti-Castro M, Vasconcellos LS, Madeira M, Soares LM, Borba VZC, Silva BCC, Moreira CA. Posicionamento Oficial da Sociedade Brasileira de Patologia Clínica/Medicina Laboratorial e da Sociedade Brasileira de Endocrinologia e Metabologia. Intervalos de Referência da Vitamina D - 25(OH)D [Internet]. Atualização 2018 [updated 2018 jan; cited 2018 junho 11]. Available from: http://bibliotecasbpc.org.br/index. php? $=4 \& \mathrm{C}=0.2$ 